\title{
SISTEMA DE GERENCIAMENTO DE DADOS PARA ANÁLISE DE SOLOS POR ESPECTROSCOPIA MOLECULAR
}

\author{
MACHAD0, G.S.1; BAUMANN, L..; PAPPIS, C.2; LIBRELOTTO, M.2; HELFER, G. A. ${ }^{3}$ KIRST, A.4; BARBOSA, J.L.V.5; \\ SANTOS, R.0.6; SANTOS, R.B6; ZIMMER, G. F.6; LOBO, E.A7; COSTA, A.B.6,7
}

PALAVRAS CHAVE: Quimiometria. Espectrometria. Solo. Software.

\begin{abstract}
RESUMO
Atualmente a identificação da maioria dos elementos encontrados no solo possuem métodos de análise atualizados e eficientes, porém a quantificação de matéria orgânica ainda é realizada através de métodos morosos e que resultam em grande quantidade de resíduos químicos. Desta maneira, surge a necessidade de tornar essa análise mais ágil e sustentável. Nos últimos tempos, o uso da espectroscopia molecular associada à quimiometria tem se destacado na avaliação qualitativa e quantitativa de diferentes tipos de materiais. Assim, o objetivo deste trabalho foi o desenvolvimento de um software, que a partir dos espectros infravermelhos das amostras de solo, seja capaz de analisar e gerar um modelo preditivo para amostras futuras, reduzindo o tempo de análise e a geração de resíduos. 0 software foi desenvolvido num ambiente de desenvolvimento integrado (IDE, Integrated Development Environment) cuja função é reunir características e ferramentas de apoio à construção de sistemas. Para isso, utilizou-se a IDE Microsoft Visual Studio 2015, versão Profissional, com alto nível de abstração de controles e classes, incluindo bibliotecas para gráficos. Além de eficiente, o Software mostrou-se capaz de analisar amostras de forma rápida e com pequena variação de resultados gerados por métodos atuais, permitindo o armazenamento das informações coletadas para criação de sistemas de predição.
\end{abstract}

\section{DATA MANAGEMENT SYSTEM FOR SOIL ANALYSIS BY MOLECULAR SPECTROSCOPY}

KEYWORDS: Chemometrics. Soil. Spectroscopy. Software.

\begin{abstract}
Currently the identification of most of the elements found in the soil have updated and efficient methods of analysis, but the quantification of organic matter is still carried out through time-consuming methods that result in large amounts of chemical residues. In this way, the need arises to make this analysis more agile and sustainable. In recent times, the use of molecular spectroscopy associated with chemometrics has been highlighted in the qualitative and quantitative evaluation of different types of materials. Thus, the objective of this work was the development of a software that, from the molecular spectra of the soil samples, is able to analyze and generate a predictive model for future samples, reducing the time of analysis and the generation of residues. The software was developed in an integrated development environment (IDE) whose function is to gather characteristics and tools to support the construction of systems. For this, we used the Microsoft Visual Studio 2015 IDE, Professional version, with a high level of abstraction of controls and classes, including libraries for graphics. In addition to being efficient, the Software was able to analyze samples quickly and with small variation of results generated by current methods, allowing the storage of collected information to create prediction systems.
\end{abstract}

\footnotetext{
${ }^{1}$ Acadêmico do Curso de Ciência da Computação da Universidade de Santa Cruz do Sul.

2 Acadêmica do Curso de Química da Universidade de Santa Cruz do Sul.

${ }^{3}$ Departamento de Computação da Universidade de Santa Cruz do Sul.

${ }^{4}$ Departamento de Química e Física da Universidade de Santa Cruz do Sul.

5 Programa de Pós-Graduação em Computação Aplicada, Universidade Vale do Rio dos Sinos.

6 Programa de Pós-Graduação em Sistemas e Processos Industriais da Universidade de Santa Cruz do Sul.

7 Programa de Pós-Graduação em Tecnologia Ambiental, Universidade de Santa Cruz do Sul.
} 


\section{INTRODUÇÃO}

Com o avanço contínuo da tecnologia e da Agricultura de Precisão (AP) é cada vez maior a procura por análises de solos. Conhecendo as condições de fertilidade e nutrição é possível fazer adubações mais efetivas e reduzir gastos. A matéria orgânica é composta por todos os organismos presentes no solo, e tem a função de fornecer nutrientes à vegetação e geração de agregados ao juntar as partículas do solo. Além disso, influi na compactação, retenção de água e estabilidade da estrutura do solo.

Existem diversas formas de analisar a matéria orgânica como, oxidação com peróxido de hidrogênio, o método de titulação Walkey-Black, onde ocorre a oxidação com dicromato de potássio em ácido sulfúrico e o método de perda por ignição. No entanto, estes são métodos morosos e caros (YARON et al., 2018). Assim, surge a necessidade do desenvolvimento de novas tecnologias com menor custo de análise e respostas mais rápidas em relação à qualidade do solo.

Dentre as metodologias que vêm sendo desenvolvidas, cabe destacar o uso da espectroscopia no infravermelho. Onde através desta técnica, é possível obter espectros que caracterizam a amostra, gerando dados rapidamente e com gastos significativamente menores do que aqueles utilizados no método referência. Além disso, trata-se de um método sustentável, sem necessitar da utilização de reagentes químicos (SANTOS, 2010).

A espectroscopia no infravermelho é um tipo de espectroscopia de absorção, onde a energia absorvida se encontra na região do infravermelho. Pode ser utilizada para identificar um composto ou identificar a composição de uma amostra (FERREIRA, 2015). A espectroscopia no infravermelho descreve a interação da radiação eletromagnética com a matéria, na região entre 660 a 4.000 nm (BARBOSA, 2007).

Recentemente, a utilização da espectroscopia no infravermelho associada à quimiometria tem se destacado, como uma ferramenta importante no desenvolvimento de metodologias analíticas inovadoras, destinadas à avaliação qualitativa e quantitativa de diferentes tipos de materiais (FERRÃO et al., 2011; ROSSO et al., 2013; VIANA et al., 2010).

A quimiometria é capaz de relacionar as medidas realizadas em um método analítico e fornecer informações através da aplicação de métodos matemáticos e estatísticos, além de permitir a manipulação de grande quantidade de dados (FERREIRA, 2015). Na literatura, diferentes aplicações da espectroscopia associada à quimiometria são descritas com sucesso, como em amostras de petróleo e derivados (PASQUINI e BUENO, 2007), óleos vegetais e biocombustíveis (FERRÃo et al., 2011) e madeira e celulose (ROSSO et al., 2013; VIANA et al., 2010). Outros estudos já comprovaram sua eficiência na aquisição de informações qualitativas e quantitativas de amostras de solos. Nestes, os autores destacam que as metodologias desenvolvidas são rápidas, de baixo custo, não destrutivas, reduzindo a geração de resíduos, e apresentando resultados qualitativos e quantitativos equivalentes aos apresentados pelos métodos de referência (DONG et al., 2011; JANIK, MERRY e SKJEMSTAD, 1998; MOHAMED et al., 2017; NIE et al., 2017; RATHOD et al., 2016; SENA et al., 2000).

Contudo, até o momento estes conhecimentos não resultaram no desenvolvimento de um sistema capaz de integrar estes conceitos de forma a gerar um produto (equipamento) que possa ser utilizado para a análise direta de materiais.

A programação orientada a objetos é o paradigma de programação que introduz a noção de "classe" e "objeto". Classes são estruturas de dados que contêm tantos campos de dados (variáveis próprias) como seus próprios métodos (funções). Um objeto é uma instância de uma classe, declarada dentro do programa (como 
uma variável do tipo "int" é declarada, um objeto de qualquer classe criada pelo programador também o deve ser) (VIANNA, 2013).

O surgimento desta estrutura está associado à facilitação da escrita de programas de computador, tornando o processo mais intuitivo. Um programa orientado a objetos deve ser visto como um conjunto de objetos capazes de interagir entre si. Cada objeto é capaz de receber processar e enviar dados, podendo ser visto como uma "máquina independente" (VIANNA, 2013).

A abordagem de Entidade-Relacionamento é baseada no Modelo Entidade-Relacionamento que foi introduzido por Peter Pin-Shan Chen, em 1976. É um aprimoramento do modelo originalmente proposto, sendo uma das técnicas de modelagem semântica mais conhecidas e, possivelmente, uma das mais utilizadas.

Uma das principais vantagens - talvez seja o motivo maior para sua popularidade - é que além de conceitos, o modelo ainda conta com uma técnica de diagramação. Isto permite registrar e comunicar de forma simplificada os principais aspectos do projeto de banco de dados.

Diante do exposto, o objetivo do presente trabalho foi o desenvolvimento de um software para aquisição dos espectros de reflectância de amostras de solos, pré-tratamento de dados, desenvolvimento e aplicação de modelos multivariados de calibração. 0 alcance deste objetivo ampliará a capacidade de atendimento da Central Analítica da UNISC, assim melhor atendendo o setor produtivo, a partir da implantação de metodologias limpas para determinação de matéria orgânica em amostras de solos, visando à otimização do tempo e economia de gastos.

\section{MATERIAIS E MÉTODOS}

\subsection{Desenvolvimento do Software}

O software elaborado para manipulação de espectros, desenvolvimento e aplicação dos modelos de calibração foi denominada Soil Data Analysis (SDA) e gerado num ambiente de desenvolvimento integrado (IDE, Integrated Development Environment) cuja função é reunir características e ferramentas de apoio à construção de sistemas.

Para isso, utilizou-se a IDE Microsoft Visual Studio 2015 (Figura 1), versão Profissional, com alto nível de abstração de controles e classes, incluindo bibliotecas para gráficos, do pacote Microsoft.NET Framework versão 4.5. A linguagem de programação adotada foi a C\# (C-Sharp). 0 software foi construído de maneira que, depois de efetuado o login, o usuário possa utilizar as ferramentas disponíveis, como a criação de novos modelos, configurações (diretórios de dados) e predição de amostras a partir de modelos principais (Figura 2). 
Figura 1: Ilustração da IDE Microsoft Visual Studio, apresentando o editor de textos onde o código é escrito.

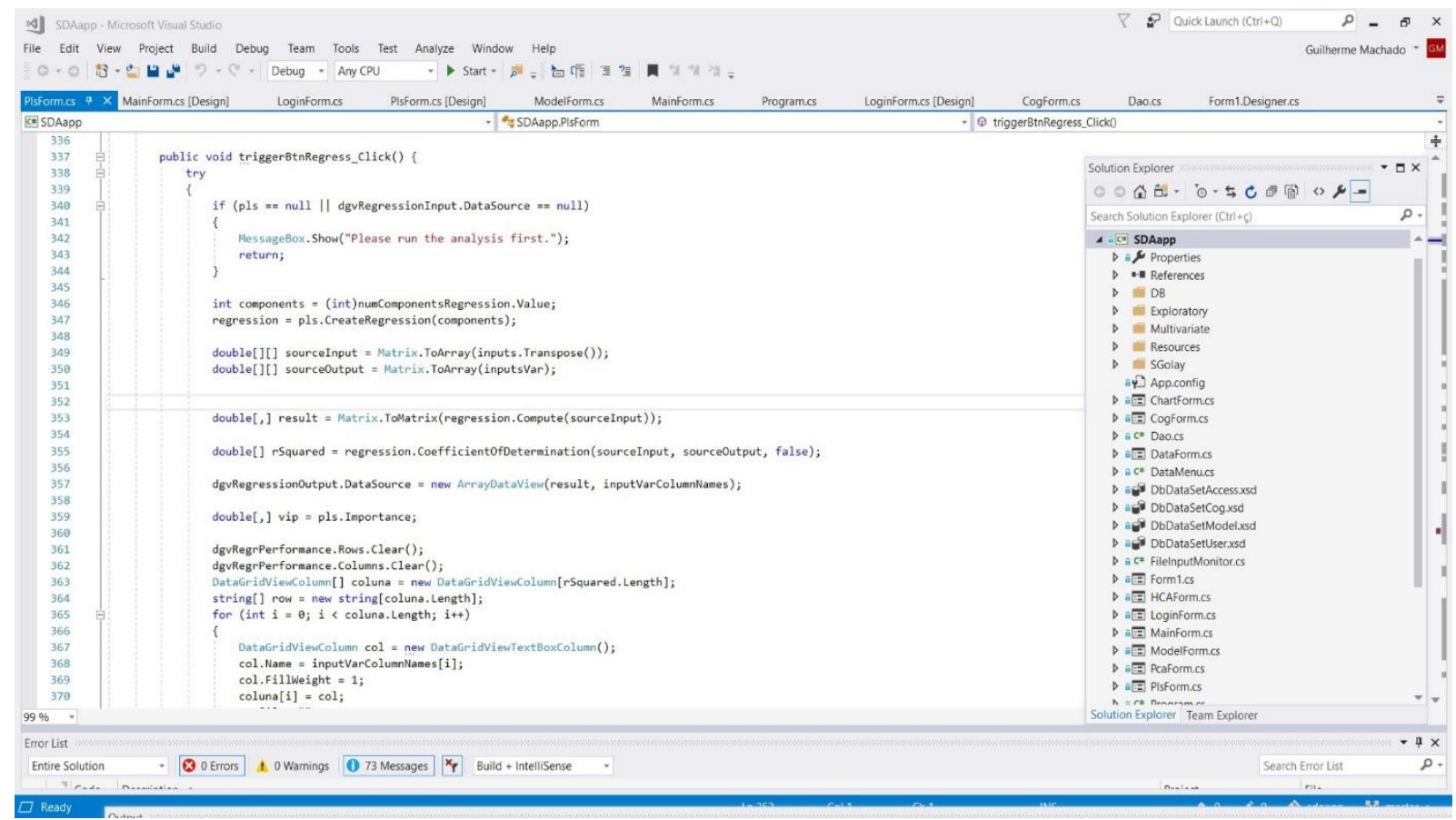

Figura 2: Menu principal do software.

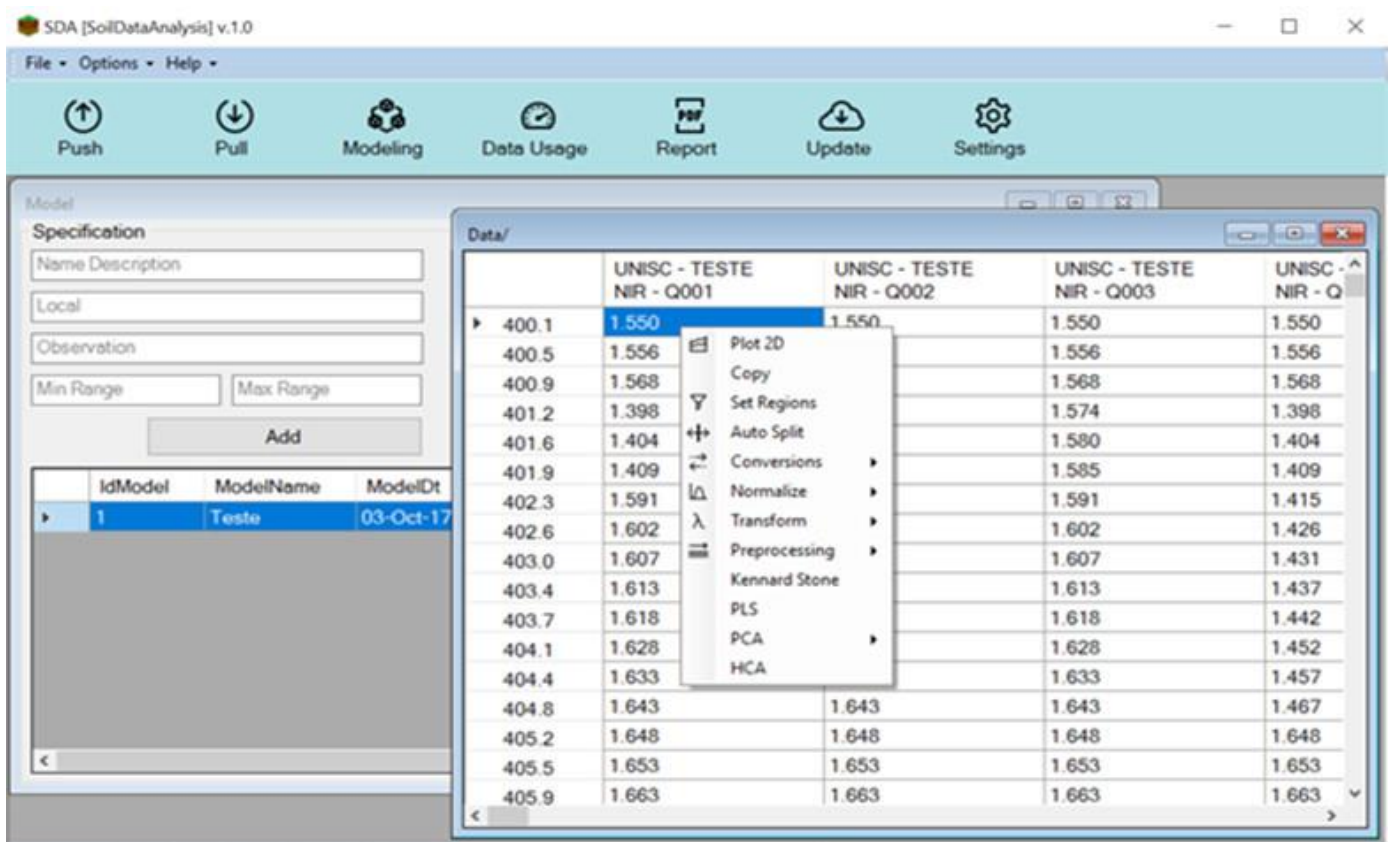

Logged gheller - Onner 0192.168 .123 (M) Connected

\subsection{Pré-tratamento de dados}

As ferramentas de pré-tratamento de dados, tem como finalidade minimizar os erros provocados por variações sistemáticas ou aleatórias, as quais não estão relacionadas às propriedades medidas, provocadas devido à instabilidade do instrumento, ao espalhamento da radiação, ou à variabilidade das propriedades físicas 
das amostras. Desta forma, foram incluídos algoritmos de correção de espalhamento multiplicativo (MSC, Multiplicative Scatter Correction), normalização, derivação (primeira e segunda derivada), e filtros de suavização (SG, Savitzky-Golay) (BEEBE, PELL e SEASHOLTZ, 1998; RINNAN, BERG e ENGELSEN, 2009).

No que se refere à calibração multivariada, foi implementado o algoritmo de regressões por mínimos quadrados parciais (PLS, Partial Least Squares) utilizando iPLS (iPLS, Interval Partial Least-Squares), com planos futuros para utilização também de siPLS (Synergy Interval Partial Least Squares) e algoritmo genético (GA, Genetic Algorithms) com ferramentas para seleção de variáveis (BEEBE, PELL e SEASHOLTZ, 1998; FERREIRA, 2015; NøRGAARD et al., 2000).

\subsection{Análises das amostras de solo}

Um conjunto de 367 amostras de solo, amostras fornecidas pela Central Analítica (UNISC), com diferentes concentrações de matéria orgânica foram utilizadas para desenvolvimento dos modelos de calibração e predição de matéria orgânica. Estas amostras foram secas em estufa modelo MA 037 (Marconi) com circulação de ar, por um período de no mínimo 24 horas, a temperatura de 45 a $60{ }^{\circ} \mathrm{C}$. Após a secagem, as amostras foram moídas em moinho de martelo modelo N1040 (Marconi), com peneira de 2 mm, e armazenadas em caixas de papelão (Tedesco et al., 1995 e EMBRAPA, 2011).

Nessas condições, a concentração de matéria orgânica nas amostras foi determinada pela metodologia de referência, seguindo as orientações descritas em Tedesco et al. (1995). A determinação de matéria orgânica no solo, baseia-se na oxidação da matéria orgânica por solução sulfocrômica em banho-maria e determinação espectrofotométrica em comprimento de onda de $645 \mathrm{~nm}$.

Para a aquisição dos espectros e desenvolvimento do método quimiométrico, as amostras passaram por uma etapa adicional de moagem e secagem. Assim, todas as amostras foram moídas novamente e peneiradas em malha de $0,5 \mathrm{~mm}$. Posteriormente, a umidade das amostras foi padronizada em estufa a $50^{\circ} \mathrm{C}$ por $24 \mathrm{~h}$. Estes procedimentos se mostraram necessários para reduzir efeitos de espalhamento de luz provocado pelas partículas de solo durante o processo de aquisição dos espectros, resultando em uma maior repetibilidade dos resultados.

Nessas condições, aproximadamente $50 \mathrm{~g}$ de cada amostra foram transferidas para frascos de poliestireno de $75 \mathrm{~mL}$, e dispostas em sistema auto-amostrador (X,Y,Z) (Figura 3), equipado com fibra ótica e sensor ótico para ajuste automático do eixo Z (distância do objeto).

A Figura 3 apresenta um conjunto de amostras durante o processo de aquisição dos espectros. Os numerais em azul e verde indicam as linhas e colunas, respectivamente, onde são posicionadas as amostras para aquisição dos espectros. Importante observar, que a posição 1 (linha 1, coluna 1) foi destinada para uma amostra padrão de refletância difusa (Spectralon $®)$. Além disto, recomenda-se que as demais posições da coluna 1 sejam utilizadas para amostras de referência de programas de controle de qualidade. A aquisição dos espectros na região entre 450 e $2.400 \mathrm{~nm}$ foi realizada diretamente sobre a superfície das amostras, com auxílio de sistema de espectrômetros com fibra óptica. 0 sistema atuou com uma resolução de 0,4 nm na região de 450 a $950 \mathrm{~nm}$, e 3,2 nm na região de 951 a $2.400 \mathrm{~nm}$, área de varredura de $4 \mathrm{~cm}^{2}$ (regulável), com velocidade de 6 mostras por minuto, conforme orientações do fabricante. Foram adquiridos 5 espectros de cada amostra.

A Tabela 1 resume as condições ideais de aquisição dos espectros das amostras de solo no sistema desenvolvido neste estudo e, as demais orientações dos fabricantes dos equipamentos. 
Tabela 1. - Condições de operação para aquisição dos espectros das amostras de solo.

\begin{tabular}{lc}
\hline \multicolumn{1}{c}{ Característica } & Configuração \\
\hline Tamanho de partícula das amostras & $<0,5 \mathrm{~mm}$ \\
Padronização de umidade & $50{ }^{\circ} \mathrm{C} \mathrm{por} 24 \mathrm{~h}$ \\
Distância entre a extremidade da fibra ótica e a amostra & $1,0 \pm 0,2 \mathrm{~mm}$ \\
Número de replicatas por amostra & 5 \\
Número de varreduras por amostra & 60 \\
Número de spins por superfície de amostra & 3 \\
Área de superfície varrida & $4 \mathrm{~cm}^{2}$ \\
Pré-tratamento dos espectros & Primeira derivada \\
\hline
\end{tabular}

Além da estatística descritiva para tabulação e ilustração gráfica dos resultados, foi aplicado o teste $t$ Students, com nível de confiança de 95\%, utilizando o software Graph Pad InStat $®$ versão 3.00. As figuras de mérito do procedimento analítico, tais como limite de detecção, quantificação e faixa de trabalho, foram determinadas conforme descrito em INMETRO (2011).

Figura 3 - Imagem sistema auto-amostrador durante a aquisição dos espectros de reflectância. Os numerais em azul e verde indicam as linhas e colunas, respectivamente, onde são posicionadas as amostras.

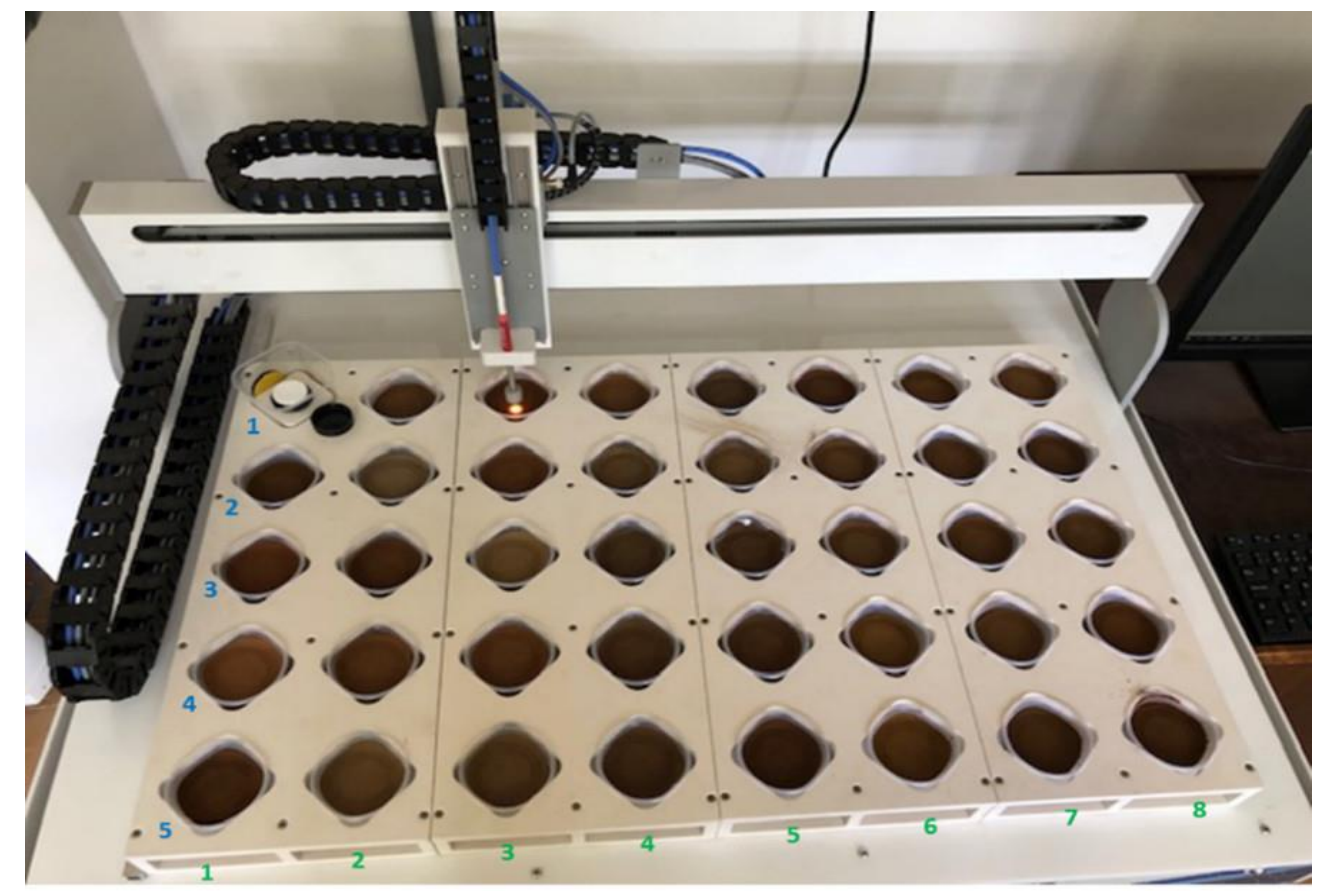

\section{RESULTADOS E DISCUSSÕES}

A interface do software desenvolvido, denominado Soil Data Analysis (SDA), está apresentada na Figura 4A, junto com o software de gerenciamento da mesa auto-amostradora na tela do computador em que está conectada. A Figura 4B apresenta a mesma tela do computador, entretanto, os softwares estão sendo executados em segundo plano, apresentando, em destaque, as notificações de resultados na tela. 
Neste exemplo estão sendo apresentados, experimentalmente, os resultados de matéria orgânica (0.M.) e argila (Clay), muito embora, até o momento apenas os resultados de matéria orgânica correspondam ao escopo deste estudo.

Estas notificações exibem os resultados de matéria orgânica imediatamente após a leitura de cada amostra, quantificando as amostras analisadas em tempo real. Além disso, é gerado um arquivo.txt com os resultados e, também os parâmetros configurados no equipamento para a análise realizada.

A quantificação de matéria orgânica por espectrometria molecular é citada na literatura por vários autores (ROSSEL et al., 2016). Entretanto, esta quantificação é feita após a aquisição dos espectros usando programas específicos de quimiometria. Portanto, essa rapidez na apresentação dos resultados é o diferencial do software desenvolvido o qual apresenta os resultados de forma instantânea.

Figura 4 - Imagens da tela do computador, conectado à mesa auto-amostradora, durante procedimento de análise de amostras de solos. (A) Tela com os softwares de gerenciamento da mesa e oSDA abertos. (B) Tela com os programas minimizados (o detalhe destaca as notificações de resultados de matéria orgânica e argila na tela).

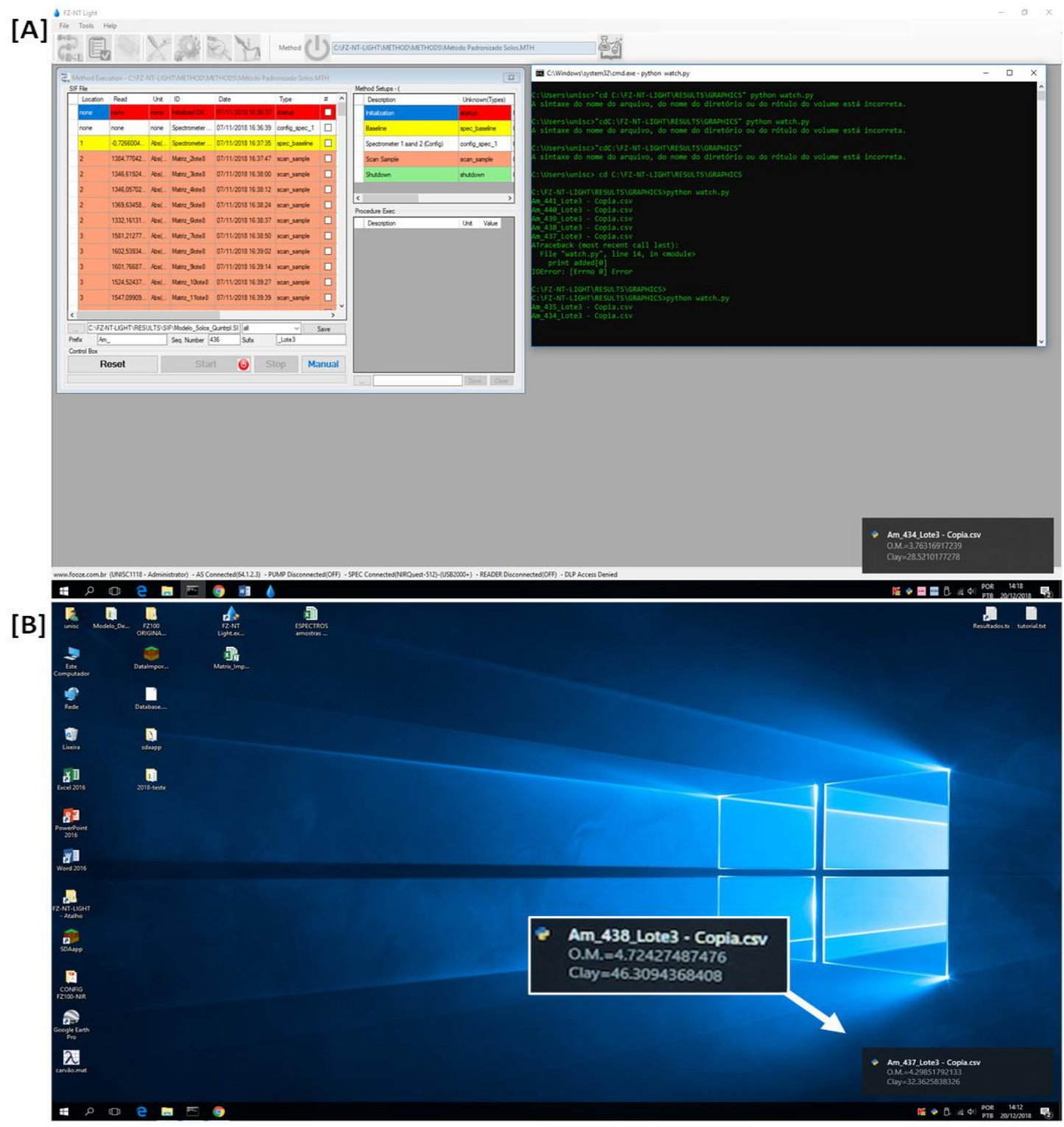


Para avaliar a eficiência do software, este foi aplicado no desenvolvimento de modelos para 367 amostras, onde os dados referentes a essas amostras foram normalizados pelo método de correção por linha de base, como pode ser visto na Figura 5. Após o pré-tratamento dos dados foi realizado o PLS. 0 modelo foi construído com 11 fatores e calibrações entre 1 a 6,7\%. 0 modelo de calibração apresentou coeficiente de determinação (R2) de 0,916, erro de calibração (RMSEC) de 0,43 e erro médio de calibração cruzada (RMSECV) de 0.50 .

Figura 5. Espectro médio ( \pm desvio-padrão) das 367 amostras na região entre 450 e $2400 \mathrm{~nm}$.

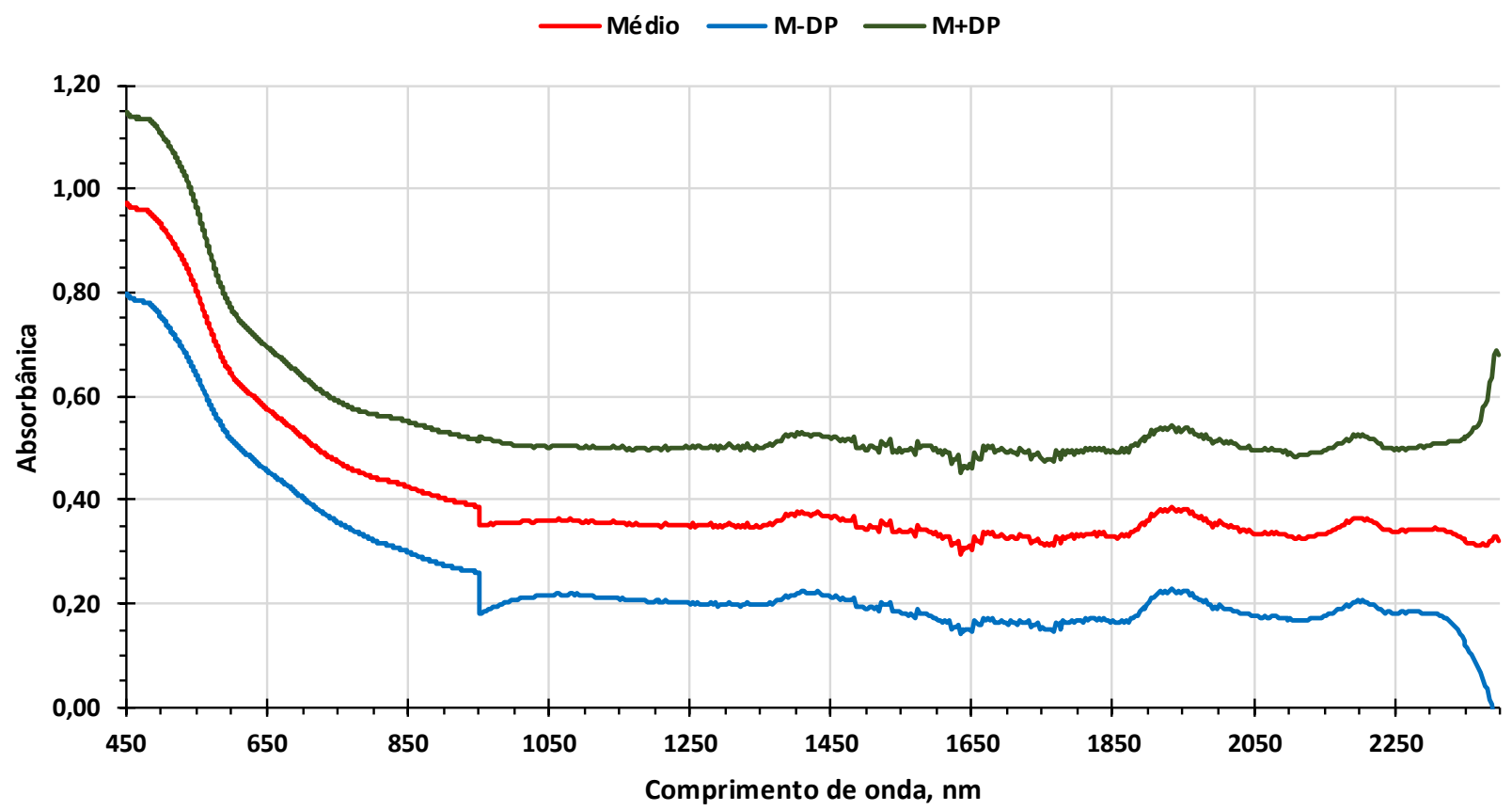

Os resultados obtidos na determinação de matéria orgânica, usando o software desenvolvido, são apresentados na Figura 6, a qual expõe os valores obtidos nos modelos desenvolvidos. Como pode ser observado na Figura 6A há uma redução significativa nos erros dos modelos até 11 variáveis latentes. A partir deste instante, a utilização de mais variáveis não agrega benefícios significativos à qualidade dos resultados. Conforme apresentado na Figura 6B, o coeficiente de determinação ( $R^{2}($ Pred)) para a determinação de matéria orgânica foi superior a 0,93 e, apresentando um erro de predição (RMSEP) de 0,41\%. Estes valores ficam entre os melhores obtidos para matéria orgânica quando se comparam com os resultados de uma série de estudos, usando a espectrometria para determinação de matéria orgânica, apresentados por ROSSEL et al., (2016), onde trabalhos relacionados de 92 países fazem parte de uma biblioteca espectral global.

Desta forma, o software desenvolvido contribui numa área onde as pesquisas, relacionadas à espectrometria, necessitam de programas que tragam agilidade e praticidade aos trabalhos. Assim como, ferramentas que possam ser adaptadas à rotina de laboratórios de análises de solos. Portanto, na continuidade dos estudos para aprimorarem o software, que apresenta um grande potencial de emprego em trabalhos relacionados, é um caminho que pode levar ao seu uso em uma gama de projetos, dependendo apenas de configurações específicas para cada necessidade. 
Figura 6. (A) Resultados dos erros de validação cruzada (RMSECV), de calibração (RMSEC), e previsão (RMSEP) em função do número de variáveis latentes utilizadas para o modelo de determinação de matéria orgânica. (B) Resultados do modelo de determinação de matéria orgânica pelo método de referência e pelo método proposto, e respectivas figuras de mérito (Amostras de calibração $=$ pontos pretos; Amostras de previsão $=$ pontos vermelhos).
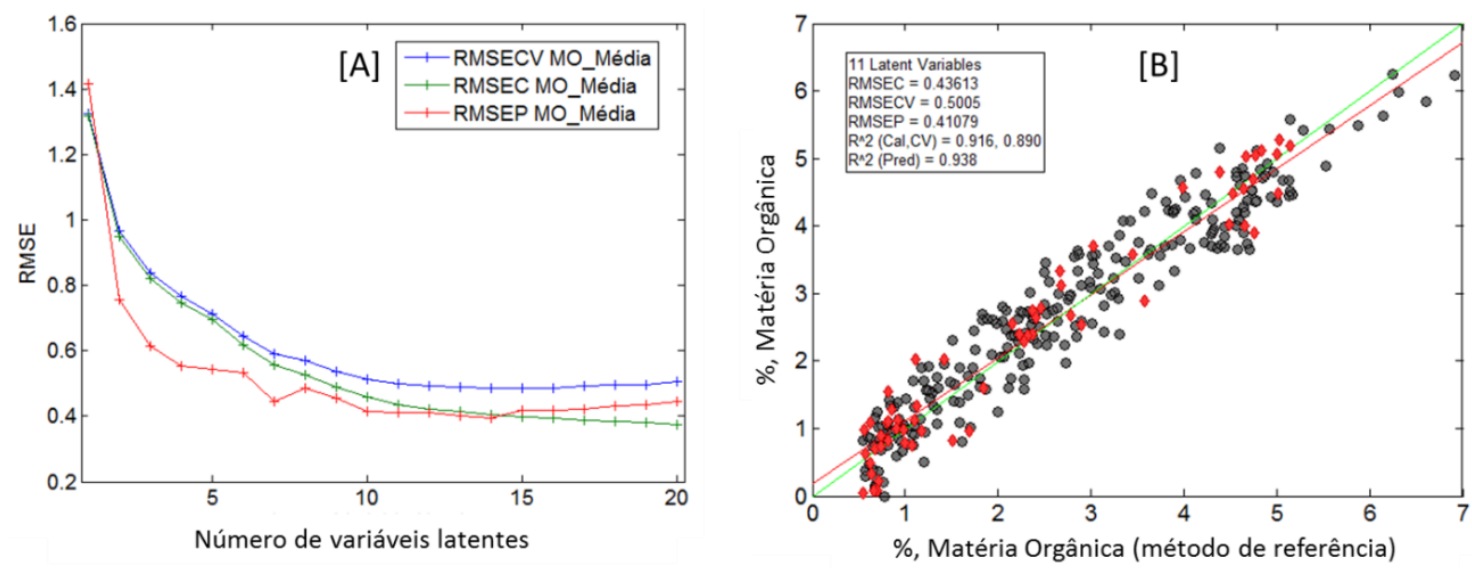

Na comparação entre os resultados obtidos pela metodologia de referência (Tedesco et al., 1995 e EMBRAPA, 2011) e a metodologia proposta neste estudo não foram identificadas diferenças significativas ( $p=$ 0,5567). A Figura 7 apresenta um gráfico comparativo entre os resultados obtidos para o conjunto de amostras de validação. Os valores de limite de detecção e quantificação foram determinados como 0,38 e 1,15\%, respectivamente. Desta forma, a metodologia proposta detecta valores de matéria orgânica a partir de 0,38\%. Contudo, resultados quantitativos devem ser considerados a partir de $1,15 \%$, perfeitamente adequado para a determinação de matéria orgânica em solos para fins agrícolas.

Figura 7: Comparativo entre os resultados de matéria orgânica obtidos pelas duas metodologias, o conjunto de amostras de validação $(n=5)$.

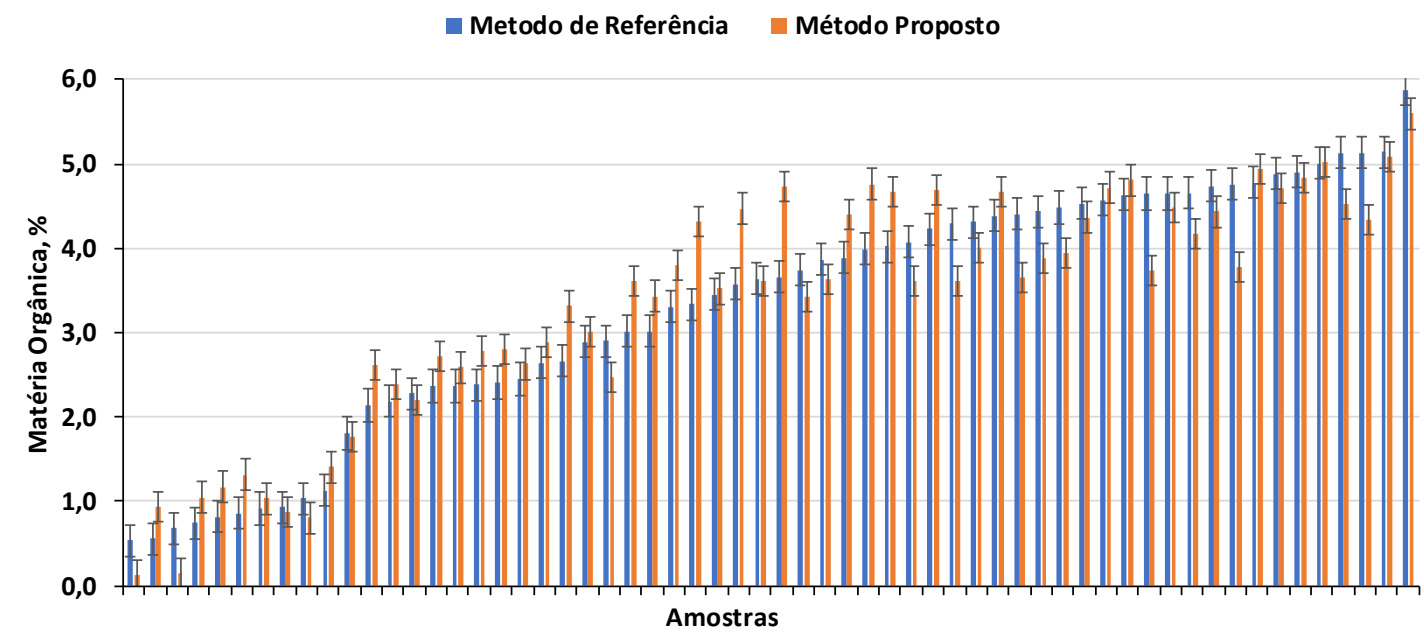

Assim, a Tabela 2 apresenta as principais figuras de mérito da metodologia desenvolvida neste estudo. 
Tabela 2. Figuras de mérito para a determinação de matéria orgânica.

\begin{tabular}{lc}
\hline \multicolumn{1}{c}{ Característica } & Matéria Orgânica \\
\hline Limite de detecção, \% & 0,38 \\
Limite de quantificação, \% & 1,15 \\
Faixa de trabalho, \% & $1,15-7,0$ \\
Erro de previsão, \% & 0,41 \\
Coeficiente de determinação (calibração) $\mathbf{R}^{2}$ & 0,916 \\
Coeficiente de determinação (previsão) $\mathbf{R}^{2}$ & 0,938 \\
\hline
\end{tabular}

Estes resultados foram comparados aos obtidos pelo software ChemoStat v2 ${ }^{\circledR}$ e Solo + Mia, e não foram identificadas diferenças significativas entre os resultados apresentados. Assim, fica comprovado o êxito do software desenvolvido, apontando-o como um promissor protagonista para os novos métodos que usam a espectroscopia molecular para quantificação de analitos em diversos tipos de matrizes, não se limitando às análises de solo.

\section{CONSIDERAÇÕES FINAIS}

Através dos resultados obtidos, conclui-se que o software desenvolvido foi satisfatório na predição de matéria orgânica. 0 software apresentou capacidade e agilidade no processo de coleta e processamento de dados, possibilitando a construção de modelos consistentes, os quais serão usados em amostras futuras, assim como também, na predição de amostras recém-analisadas, as quais passam pelos modelos de matéria orgânica e argila e já tem seus valores exibidos para o usuário.

Com tamanha agilidade no tratamento de dados, juntamente com a automação da predição após análise, amostras distintas que apresentam resultados atípicos podem ser rapidamente identificadas, sendo refeitas ou deixadas sob vigilância. Assim, o software se mostrou promissor perante o objetivo de aumentar a produtividade analítica, colaborando no aperfeiçoamento de métodos que visam reduzir a grande quantidade de resíduos tóxicos utilizados pelos métodos convencionais, além de apresentar consistência e acurácia em seus resultados.

\section{AGRADECIMENTOS}

Os autores agradecem à Secretaria de Desenvolvimento Econômico, à Ciência e Tecnologia do Estado Rio Grande do Sul, à Central Analítica da UNISC, ao Conselho Nacional de Desenvolvimento Científico e Tecnológico (CNPq), à Fundação de Amparo à Pesquisa do Estado do Rio Grande do Sul (FAPERGS) e a Coordenação de Aperfeiçoamento de Pessoal de Nível Superior (CAPES).

\section{REFERÊNCIAS}

BARBOSA, Luiz Cláudio de Almeida. Espectroscopia no infravermelho na caracterização de compostos orgânicos. Viçosa: Universidade Federal de Viçosa, 2007 
BARROS NETO, Benício de; SCARMINIO, leda S.; BRUNS, Roy E. 25 anos de quimiometria no Brasil. Química Nova, v. 29,p. 1401-1406. 2006.

BEEBE, Kenneth R.; Pell, Randy J.; Seasholtz, Mary Beth. Chemometrics: A pratical guide. New York: John Wiley \& Sons, Inc., 1998. Disponível em: http://www.wiley.com/WileyCDA/WileyTitle/productCd-0471124516.html.

DONG, YI-WEI et al. Determination of Soil Parameters in Apple-Growing Regions by Near- and Mid- Infrared Spectroscopy. Pedosphere, v. 21, n. 5, p. 591-602, 2011/10/01/2011.

EMBRAPA - Agricultura de precisão: resultados de um novo olhar. Manual de métodos de análise de solos, 2011. $230 \mathrm{p}$.

FERRÃO, MARCO FLÔRES et al. Simultaneous determination of quality parameters of biodiesel/diesel blends using HATR-FTIR spectra and PLS, iPLS or siPLS regressions. Fuel, v. 90, n. 2, p. 701-706, 2011/02/01/2011.

FERREIRA, M. M. C.; Quimiometria-Conceitos, Métodos e Aplicações, Campinas, SP: Editora Unicamp, Vol., n., p., 2015.

JANIK, L. J.; MERRY, R. H.; SKJEMSTAD, J. O. Can mid infrared diffuse reflectante analysis replace soil extraction? Australian Journal of Experimental Agriculture, v. 38, n. 7. p. 681-696, 1998.

MICROSOFT, VISUAL STUDIO. Visual Studio Professional 2015: Microsoft 2015.

MOHAMED, E. S. et al. Application of near-infrared reflectante for quantitative assessment of soil properties. The Egyptian Journal of Remote Sensing and Space Science, 2017/02/20/ 2017.

NIE, PENGCHENG et al. Detection of Soil Nitrogen Using Near Infrared Sensors Based on Soil Pretreatment and Algorithms. Sensors (Basel), v. 17, n. 5, 2017.

NORGAARD, I. et al. Interval Partial Least-Squares Regression (iPLS): A Comparative Chemometric Study with an Example from Near-Infrared Spectroscopy. Applied Spectroscopy, v. 54, n. 3, p. 413-419, 2000.

PASQUINI, CELIO; BUENO, AERENTON FERREIRA. Characterization of petroleum using near-infrared spectroscopy: Quantitative modeling for the true boiling point curve and specific gravity. Fuel, v. 86, n. 12, p. 1927-1934, 2007/08/01/ 2007.

RATHOD, PARES $\mathrm{H}$. et al. Analysis of visible and near infrared spectral reflectance for assessing metals in soil. Environ Monit Assess, v. 188, n. 10, p. 558, September 102016.

RINNAN, A.; Van Den Berg, F.; Engelsen, S. B. Review of the most common preprocessing techniques for nearinfrared spectra. Trends in Analytical Chemistry, v. 28, n. 10, p. 1201-1222, 2009.

RINNAN, A.; VAN DEN BERG, F.; ENGELSEN, S. B. Review of the most common preprocessing techniques for nearinfrared spectra. Trends in Analytical Chemistry, v. 28, n. 10, p. 1201-1222, 2009.

RINNAN, ÅSMUND; BERG, FRANS VAN DEN; ENGELSEN, SØREN BALLING. Review of the most common preprocessing techniques for near-infrared spectra. TrAC Trends in Analytical Chemistry, v. 28, n. 10, p. 1201-1222, 2009/11/01/ 2009.

ROSSEL, R.V. et al. A global spectral library to characterize the world's soil. Earth-Science Reviews, 155, p. 198230, 2016. DOI: https://dx.doi.org/10.1016/j.earscirev.2016.01.012.

ROSSO, SILVIANA et al. Estimate of the density of Eucalyptus grandis W. Hill ex Maiden using near infrared spectroscopy. CERNE, v. 19, p. 647-652, 2013.

SANTOS, G.A.; PEREIRA, A.B. \& KORNDÖRFER, G.H. Uso do sistema de análise por infravermelho próximo (NIR) para análise de matéria-orgânica e fração de argila em solos e teores foliares de silício e nitrogênio em cana-deaçúcar. Biosci. J., 26:100-108, 2010.

SENA, MARCELO M. DE et al. Avaliação do uso de métodos quimiométricos em análise de solos. Química Nova, v. 23, p. 547-556, 2000. 
TEDESCO, M. J.; GIANELLO, C.; BISSANI, C. A.; BOHNEN, H; VOLKWEISS, S. J. Análise de solo, plantas e outros materiais. $2^{\circ}$ ed. Ver. e Ampl. - Porto Alegre UFRGS Departamento de Solos, 1995. p. 174.

VIANNA, J. T. A. Introdução à orientação a objetos, 2013. Disponível em: < http://www.ufjf.br/peteletrica/files/2012/10/Curso-Orienta\%C3\%A7\%C3\%A3o-a-objetos.pdf >. Acesso em: 10 de março de 2019.

YARON, O. et al. Evaluating the detection limit of organic matter using point and imaging spectroscopy. Revista Geoderma. p. 100-109, 2018. DOI: https://doi.org/10.1016/j.geoderma.2018.02.011. 